\title{
Cutaneous Field Cancerization on a Vitiligo Area
}

Luciana Falivene Cará*, Julia de Ávila Fowler, André Cesar Antiori Freire Pessanha, Denise Steiner and Felipe Ribeiro da Silva

Department of Dermatology, University of Mogi das Cruzes, Mogi das Cruzes, Brasil

*Corresponding author: Luciana Falivene Cará, Department of Dermatology, University of Mogi das Cruzes, Mogi das Cruzes, Brasil, Tel: +55 12 997340810; E-mail: lucianafcara@yahoo.com.br

Received date: March 30, 2017; Accepted date: April 17, 2017; Published date: April 23, 2017

Copyright: (C) 2017 Cará LF, et al. This is an open-access article distributed under the terms of the Creative Commons Attribution License, which permits unrestricted use, distribution, and reproduction in any medium, provided the original author and source are credited.

\begin{abstract}
Vitiligo is the most commom depigmentary disorder of the world, with probably an autoimmune cause. Studies reveal the presence of genes likely the emergence of Vitiligo, many of them involved in the synthesis of proteins related to the recognition and killing of melanocytes. The interaction of these genes may suggest protection and good prognosis for Vitiligo patients in relation to skin cancer. We describe the case of a patient with field cancerization in areas affected by Vitiligo, counteracting, therefore, the usual.
\end{abstract}

Keywords: Vitiligo; Skin neoplasms; Hypopigmentation; Pigmentation disorders; Pigmentation; Skin pigmentation; Melanoma

\section{Introduction}

Vitiligo is the most commom depigmentary disorder of the world. The oldest reports of a disease similar to Vitiligo known until today date from $1500 \mathrm{AC}$, present in sacred Hindu writings and in texts of ancient Egypt [1]. Classified as a disease of acquired character, in the most cases, with a prevalence of $0,5 \%$ all around the world [2], without predilection of gender, racial or age [3-5] although some studies reveal prevalence in females. Clinically, is expressed by patches and macules, achromic and rarely hypochromic, located mainly in the skin and mucous membranes, due to the decrease or absence of melanocytes, resulting from the autoantibodies produced by the own body that will eventually destroy them. These cells, besides granting the skin color through the melanin production, protect it from the effects of solar and chemicals carcinogens [6]. Recently, studies reveal the presence of susceptible genes related to the appearance of Vitiligo, being a large part of them, involved in protein synthesis related to the recognition and death of melanocytes. The interaction of these genes suggests protection and good prognosis to patients with Vitiligo in relation to skin cancer [7].

The occurrence of malignant melanoma in skin areas affected by Vitiligo is extremely rare, since it challenges the antagonistic relationship between these two pathologies.

It is reported a case of cutaneous field canceritazion in Vitiligo.

\section{Case Report}

We report on a 71-year-old female, housewife, brasilian, born and resident in the interior of Sao Paulo state, patient referenced to this service reporting red and scaly lesions all over the body 5 years ago. States that 15 years ago white spots appeared, initially in face and subsequently spreading to the rest of the body, being diagnosed with Vitiligo. In the dermatological examination, she presented disseminated achromic macules, with few not affected skin areas, besides small erythematous crusted plates lesions over her left forearm (Figures 1-3), face, back of hands and dorsum of the feet (sun-exposed regions). The initial hypothesis was generalized Vitiligo and actinic keratosis (KA). After surgical remove of some lesions, it was given the diagnosis by histopatholoy biopsy of: actinic keratosis with associated folliculitis, squamous cell carcinoma moderately differentiated in the left eyelid treated surgically by Mohs micrographic surgery.

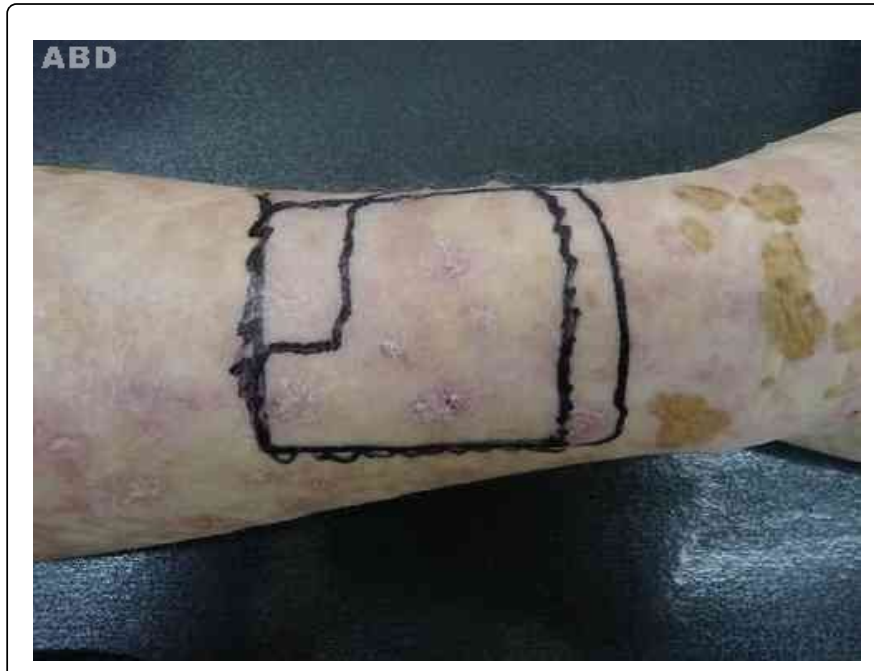

Figure 1: Small erythematous crusted plates lesions on left forearm. 


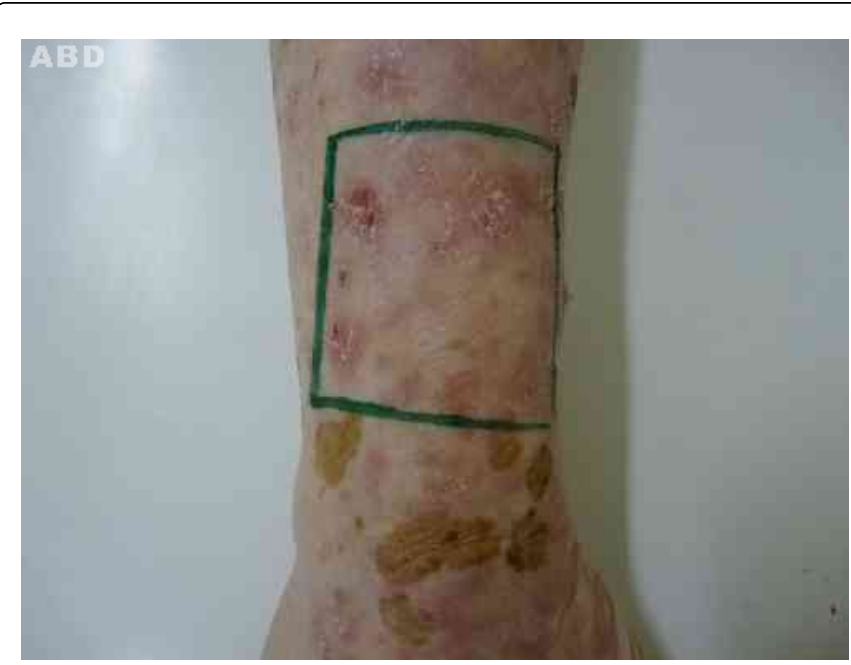

Figure 2: Expanded vision of lesions, allowing visualization of flaking and redness.

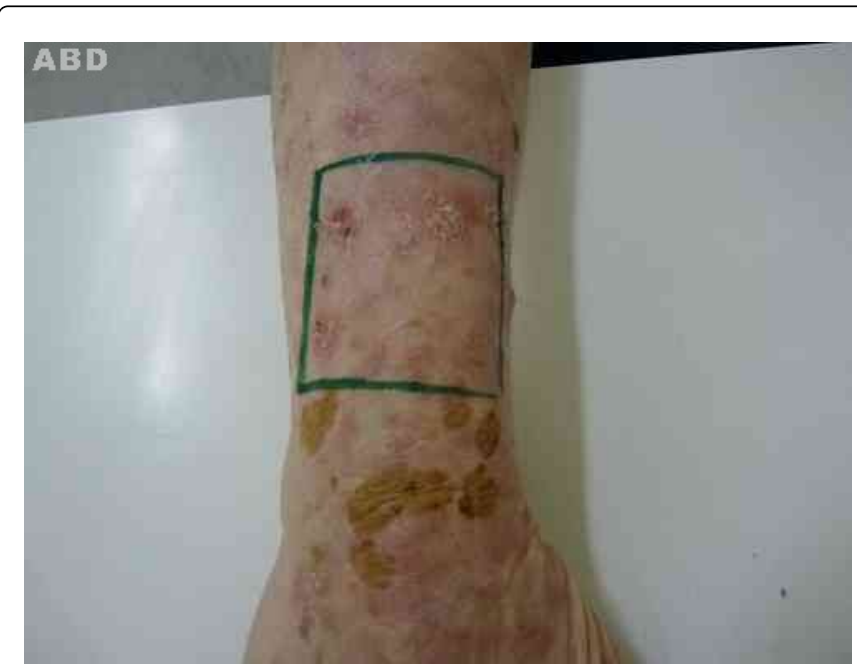

Figure 3: Lesions in the left forearm measuring about $2 \mathrm{~cm}$.

\section{Discussion}

Studies report that there are genes that confer susceptibility to Vitiligo. Most of them encodes immunoregulatory proteins, especially to the pathways wich melanocytes can be recognized and killed. Besides that, there is an association between Vitiligo and polymorphism in the gene TYR, wich encodes tyrosinase, enzyme involved in melanin synthesis. Discrete allelic interactions in these genes related to different immunological recognition of tyrosinase in Vitiligo and melanoma, suggest that the intense expression of antityrosinase protects patients with Vitiligo against melanoma. This gene interaction may also explain the frequent occurrence of Vitiligo in patient treated for melanoma, in wich the development of autoimmune disorder is a sign for positive prognosis, conferring increased survival of this patients [7].

Teulings et al. [5] analyzing 1.307 patients with and without Vitiligo noted that the probability of developing skin cancer in patients with Vitiligo it was considerably low. Besides that, among patients with Vitiligo that developed skin cancer, only 2 of them had basal cell carcinoma in Vitiligo's affected area, illustrating this protection [6].

There are many mechanisms that attempt to explain the protection Vitiligo confers against skin cancer. Between then: patients with Vitiligo protect the skin more often from sun exposure [7-9]; the anti melanocytic autoimmune response of Vitiligo 10; theoretical impossibility of melanoma arising in the areas affected by Vitiligo, since the melanocytes were destroyed [6]; Vitiligo's patients seem to have overexpression of $\mathrm{p} 53$, tumor suppressor gene, wich possibly explains the low risks for CBC and CEC7 [10]; due to autoimmune mechanism, Vitiligo patients have overproduction of proinflammatory cytokines IL- 1 and TNF- $\alpha$, that stimulates the production of superoxide dismutase and glutathione peroxidase, reducing the risk of skin cancer [10].

A research was made in Bireme and Scielo database, being found so far 10 case report and studies with large samples, between the years of 1991 and 2014 , in wich Vitiligo preceded the skin cancer, highlighting the rarity of this study and the importance of it to the scientific community.

\section{References}

1. Tarlé RG, Nascimento LM, Mira MT, Castro CC (2014) Vitiligo - Part 1. An bras dermatol 89: 461-470.

2. Hammoud S, Kruis R, Sigurdsson V (2016) Prediction of the Occurrence of Melanoma and Non-melanoma Skin Cancer in Patients with Vitiligo. Acta Dermato Venereologica 96: 106-107.

3. Nordlund J (2006) the pigmentary system. 2nd ed. Oxford (UK): Blackwell Publishing.

4. Paradisi A, Tabolli S, Didona B, Sobrino L, Russo N, et al. (2014) Markedly reduced incidence of melanoma and nonmelanoma skin cancer in a nonconcurrent cohort of 10,040 patients with Vitiligo. J Am Acad Dermatol 71: 1110-1116.

5. Teulings HE, Overkamp M, Ceylan E, Nieuweboer-Krobotova L, Bos JD, et al. (2012) Decreased risk of melanoma and nonmelanoma skin cancer in patients with Vitiligo: a survey among 1307 patients and their partners. Br J Dermatol 168: 162-171.

6. Lindelöf B, Hedblad MA, Sigurgeirsson B (1998) On the Association Between Vitiligo and Malignant Melanoma. Acta Derm Venereol 78 : 483-484.

7. Sandoval-Cruz M, García-Carrasco M, Sánchez-Porras R, Mendoza-Pinto C, Jiménez-Hernández M, et al. (2011) Immunopathogenesis of Vitiligo. Autoimmun Rev 10: 762-765.

8. Schallreuter K, Tobin D, Panske A (2002) Decreased Photodamage and Low Incidence of Non-Melanoma Skin Cancer in 136 Sun-Exposed Caucasian Patients with Vitiligo. Dermatology 204: 194-201.

9. Feily A, Pazyar N (2011) Why Vitiligo is associated with fewer risk of skin cancer? Providing a molecular mechanism. Arch Dermatol Res 303: 623-624.

10. Spritz R (2010) The genetics of generalized Vitiligo: autoimmune pathways and an inverse relationship with malignant melanoma 2: 78. 